\title{
Reduction of Clathrate Hydrate Film Growth Rate by Naturally-Occurring Surface Active Components
}

\author{
Shane A. Morrissy, Angus J. McKenzie, Brendan F. Graham, \\ Michael L. Johns, Eric F. May, Zachary M. Aman*

\begin{abstract}
Fluid Science and Resources Division, School of Mechanical and Chemical Engineering, The University of Western Australia, 35 Stirling Highway, Crawley, Western Australia 6009, Australia
\end{abstract}

\begin{abstract}
During the production of offshore oil and gas, the cooling of hydrocarbons toward seafloor temperatures enables the formation of gas hydrates, which may restrict fluid flow and ultimately block the flowline. During the formation of a hydrate blockage, a hydrate film may grow between individual particles or between hydrate particles and the pipeline wall, respectively resulting in a higher slurry viscosity or a reduced hydraulic diameter. This hydrate film growth rate has been previously studied as a function of pressure, temperature, and hydrate guest species, but limited data are available to guide whether naturally-occurring surface active components in the oil may affect the hydrate film growth rate. In this study, we have used a micromechanical force (MMF) apparatus to quantify the film growth rate of cyclopentane hydrate at moderate subcooling. Naturally-occurring surface active species were obtained from an Australian crude oil by solvent extraction to separate out asphaltenes, binding resins, and free resins. Each crude oil fraction was then added to a chemically inert hydrocarbon phase, and the impact of the hydrate film growth rate was measured. The results illustrate that, at mass fractions below $300 \mathrm{ppm}$, the hydrate film growth rate was reduced by at least one order of magnitude with asphaltenes and free resins being the most and least effective fractions, respectively, at suppressing hydrate film growth rate. The presence of each fraction also caused an increase in the wetting angle of the water droplet on the hydrate particle surface, which suggested that these naturally-occurring components may adsorb to the hydrate particle surface. Measurements with the MMF revealed that each of the three fractions was able to reduce the hydrate particle cohesive force by two orders of magnitude.
\end{abstract}




\section{Introduction}

The production of deepwater offshore oil and gas assets typically requires long subsea tiebacks connected to a central processing facility. In some cases, high-pressure hydrocarbon fluids can cool to seafloor temperatures within the residence time of the flowline. ${ }^{1}$ Under these conditions, clathrate hydrates become thermodynamically stable, where molecular cages of water form around light hydrocarbon species to form ice-like crystalline solids. ${ }^{1,2}$ If hydrate formation in oil or gas pipelines is not fully suppressed through the addition of antifreeze thermodynamic hydrate inhibitors (THIs), the resultant hydrate particles may aggregate together or deposit along the flowline wall, restricting or ultimately blocking the transit of hydrocarbon fluids. ${ }^{3}$ The removal of hydrate blockages is a high-cost and high-risk operation, which may be achieved through depressurization, flowline heating ${ }^{4}$, and/or the injection of THIs. ${ }^{5}$

Beyond their relevance to conventional energy systems, clathrate hydrates also occur in nature due to biogenic or thermogenic methane release near the ocean floor or under the permafrost ${ }^{6}$ the total hydrocarbon content stored in natural hydrate formations has been estimated to be larger than that of any other fossil fuel. ${ }^{6,7}$ Hydrate-bearing sands are of particular interest, ${ }^{8}$ where the production of methane may be controlled by thermodynamic destabilization. ${ }^{9}$ The sustained recovery of methane from hydrated sediments has yet to be achieved on a commercial scale, due largely to uncertainties in how hydrate particles interact with, and grow along, the pore boundaries..$^{10,11}$

In both conventional and alternative energy applications, engineers require an understanding of the hydrate film growth rate in both chemically inert and contaminated environments. For the past three decades, hydrate growth rates have been studied as a bulk property; Vysniauskas and Bishnoi reported the growth rate of methane ${ }^{12}$ and ethane ${ }^{13}$ hydrates in a stirred tank, delivering fundamental information about the kinetic rate constants. Englezos et al. ${ }^{14}$ extended this understanding to include methane-ethane gas mixtures; these datasets were adapted by Sloan, Koh and co-workers to predict hydrate growth rate in multiphase pipelines using the OLGA transient multiphase flow simulator. ${ }^{15}$

Much of the understanding about hydrate growth rate is based on macroscopic scale experiments. Limited measurements of hydrate growth rate along a water-hydrocarbon 
interface, particularly in the presence of surfactants, are available in the literature. This understanding is important when assessing the severity of potential hydrate blockages in oil and gas pipelines, where such film growth acts to (i) sinter existing hydrate particles together ${ }^{16}$ and (ii) allow for a solid crystalline layer to grow directly along the flowline wall. ${ }^{17}$ Cohesive force measurements have illustrated that the strength of a hydrate aggregate increases substantially when particles grow together, ${ }^{18}$ and the force of hydrate adhesion to the pipeline wall can increase by at least two orders of magnitude if a hydrate film grows between the particle and steel wall. ${ }^{19}$

Measurements by Uchida et al. ${ }^{20}$ reported the rate of $\mathrm{CO}_{2}$ hydrate film growth at the interface of a water droplet, where dendritic-type growth was observed after the initial film formation; the authors proposed that the film growth rate in that experiment was limited by the diffusion of heat away from the hydrate particle. ${ }^{20}$ Mori ${ }^{21}$ applied a convective heat transfer model to predict the initial film thickness of a $\mathrm{CO}_{2}$ hydrate, which did not accurately reproduce estimates of film thickness reported by Uchida et al. ${ }^{20}$ due to limitations in capturing lateral thickness variations. ${ }^{21}$ Uchida et al. ${ }^{22}$ later noted that subcooling from the hydrate phase boundary was a critical parameter in assessing $\mathrm{CO}_{2}$ hydrate film growth rate, and further noted that the addition of sodium chloride $(\mathrm{NaCl})$ to the aqueous phase reduced hydrate growth rate proportional to brine salinity; the authors hypothesized that $\mathrm{NaCl}$ buildup at the interface imposed a mass transport limitation, as electrolyte species must be rejected from the growing crystal lattice. ${ }^{22}$

Taylor et al. ${ }^{23}$ reported the growth rate of methane and cyclopentane hydrate films, and also observed that the initial film thickness scaled directly with both subcooling ${ }^{20,22}$ and the solubility of the hydrate guest species in water. While methane hydrate films had an initial thickness of $5 \mu \mathrm{m}$ that thickened to between 20 and $100 \mu \mathrm{m}$ during the observation period, cyclopentane hydrate films had an initial thickness of $12 \mu \mathrm{m}$ that grew to between 15 and 35 $\mu \mathrm{m}$. Saito et al. ${ }^{24}$ reviewed film growth rate data as a function of subcooling and modelled them in terms of the solubility of the guest molecule in water. They concluded that both mass and heat transport resistances were critical to the prediction of hydrate film growth.

More recently, Wu et al. ${ }^{25}$ measured the growth of methane-propane hydrate films exposed to LuviCap EG, a commercial kinetic hydrate inhibitor (KHI). ${ }^{26}$ By measuring the lateral film growth rate and applying the heat transfer-limited growth model from Mori, ${ }^{21} \mathrm{Wu}$ et al. ${ }^{25}$ 
calculated an initial film thickness in their baseline measurements that was consistent with values measured by Taylor et al. ${ }^{23}$ The presence of the KHI reduced both the growth rate and thickness of the hydrate film, suggesting the interaction of KHI with the water-oil or hydrateoil interfaces introduced additional mass transport limitations. Wu et al. ${ }^{27}$ later extended this investigation to cyclopentane hydrate, reporting a two order-of-magnitude reduction in film growth rate when the KHI PVCap was added in the system.

However, while previous studies have focused on whether KHIs affect hydrate film growth rate, there are few available data to suggest whether natural or synthetic surfactants found in crude oil pipelines may share similar properties, including corrosion inhibitors, demulsifiers, KHIs and hydrate anti-agglomerants (AAs). ${ }^{28}$ Zhong and Rogers ${ }^{29}$ reported that sodium dodecyl sulfate (SDS) promoted the rapid growth of hydrates with the growth rate increasing by up to 700 times. Insufficient evidence was presented to elucidate the mechanism behind this behavior because, as Di Profio et al. ${ }^{30}$ noted, the SDS systems studied by Zhong and Rogers were below the critical micelle concentration (CMC). Di Crescenzo et al. ${ }^{31}$ also observed that anionic surfactants with sulfur-containing head groups were highly effective at promoting hydrate growth, but there remained uncertainty as to the molecular mechanism responsible for such behavior. Di Crescenzo et al. ${ }^{31}$ hypothesized that surfactant adsorption to the water-oil interface may enhance water structuring and thereby increase hydrate growth rate.

In conventional oil and gas flowlines, hydrate interfaces may be exposed to an array of surfactant species that occur naturally in crude oil. At a simple level, oil constituents may be split into four classes through a SARA (saturates, aromatics, resins, asphaltenes) fractionation, ${ }^{32}$ in which components separate based on their polarity and solubility in light alkanes. Asphaltenes and resins are the first and second most polar fractions of crude oil, respectively. Work by Graham et al. ${ }^{32}$ proposed a modification to the SARA fractionation technique based on a procedure by Ghloum and Oskui, ${ }^{33}$ where separated asphaltenes are subsequently washed with hot heptane to dissolve and collect binding resins (BRs) that dissolve in the liquid phase; the remaining non-heptane soluble solids were named residual asphaltenes (RAs). The mass ratio of BRs to RAs was shown to correlate inversely with the tightness of the water-in-oil emulsions ${ }^{33}$ formed for West African and Western Australian crude oils. ${ }^{32}$ This observation suggests that BRs may function to solvate RAs in the oil phase, which would otherwise adsorb to the water-oil interface as surfactants. Spectroscopic 
analysis of the BRs and bulk resin class - hereinafter called free resins (FRs) - identified that both the BR and FR contain straight chain hydrocarbons with terminal polar functionalities including acids, aldehydes and alcohols; the BRs possessed small polyaromatic ring systems at one end. Graham et al. ${ }^{32}$ proposed that the BRs were a subfraction of the resin class, in which the species interact strongly with asphaltenes resulting in co-precipitation.

At present, there are limited data to indicate whether these surface active species in crude oil (BRs, RAs, and FRs) may affect the growth or aggregation behavior of hydrate particles in subsea oil and gas flowlines. This study deploys a unique method to visualize and measure hydrate film growth rate in the presence of a modified hydrocarbon continuous phase, into which each crude oil subclass extracted from the same Australian crude oil was injected. This work provides new fundamental insights into the growth mechanics and particle morphology for clathrate hydrate interfaces that have been exposed crude oils with similar surface active components. 


\section{Methods and Materials}

\subsection{SARA Fractionation}

To generate the RA, BR and FR subclasses, $100 \mathrm{~g}$ of the Australian crude oil used by Graham et al. ${ }^{32}$ was first evenly proportioned into ten batches. Each batch was then diluted in $300 \mathrm{~mL}$ of analytical grade heptane (Sigma-Aldrich) at ambient temperature and atmospheric temperature, based on the procedure described by Graham et al. ${ }^{32}$ (Figure 1). The crude oil was previously characterized with an RA, BR, and FR content of $0.07,0.05$, and $2.91 \mathrm{wt} \%$, respectively, and saturates and aromatics constituted $96.97 \mathrm{wt} \%$ of the oil sample; Fridjonsson et $\mathrm{al}^{34}$ measured the oil specific gravity to be 0.9 . The water-oil emulsion stability of the oil was not investigated in this study; however, the equilibrium interfacial tension between this oil and synthetic brine $(3.5 \mathrm{wt} \% \mathrm{NaCl})$ was measured using a pendant drop tensiometer ${ }^{35}$ to be $13.7 \mathrm{mN} / \mathrm{m}$, suggesting the oil may form stable emulsions under appropriate watercut and pressure conditions. At the end of the fractionation process, 5-15 wt \% of each extracted component was added to a bottle of toluene for storage, as this solvent can dissolve all crude oil subclasses.

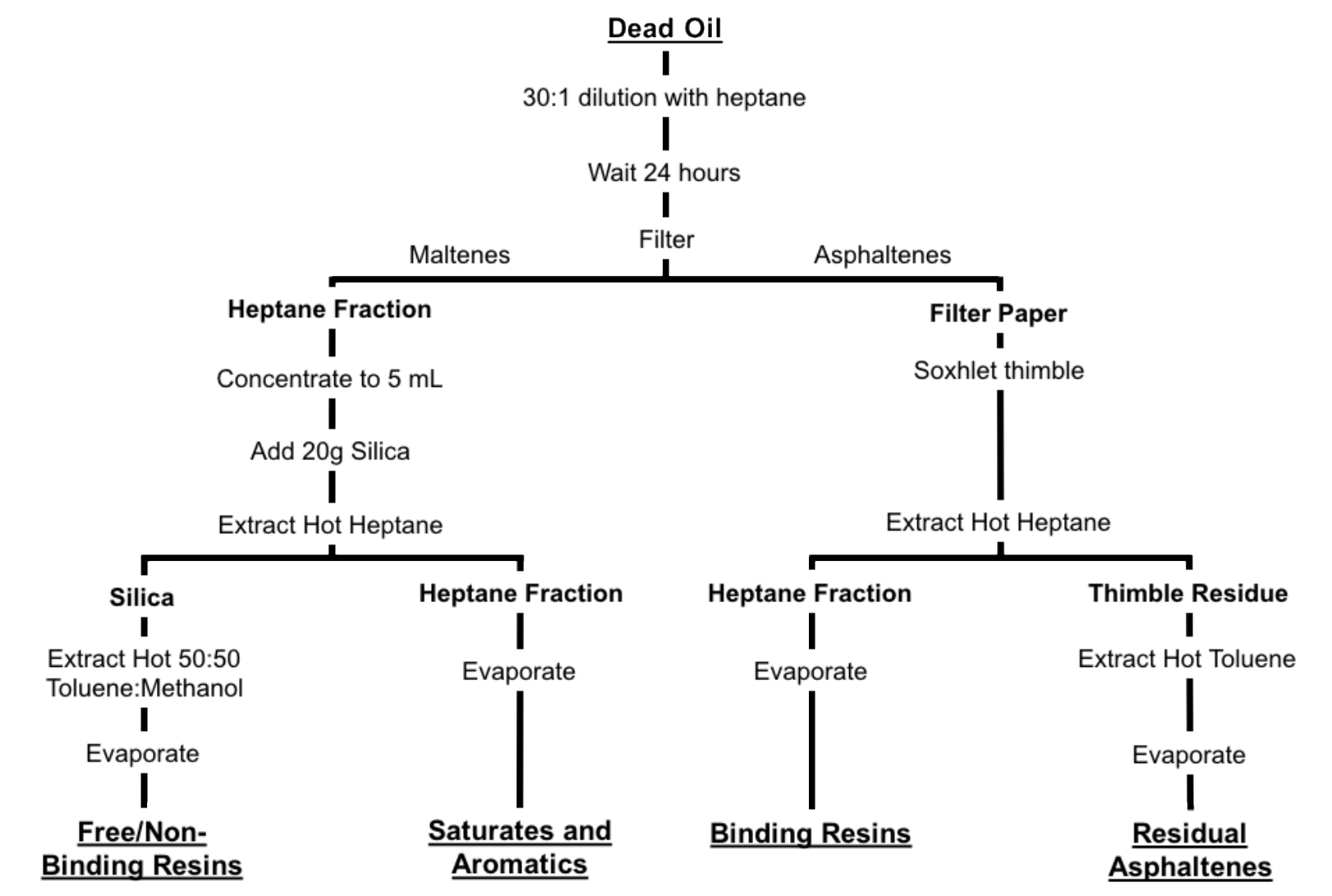

Figure 1. The extended SARA fractionation scheme for crude oil used in this work, adapted from Graham et al. ${ }^{32}$ 


\subsection{Micromechanical Cohesive Force Measurement}

A third-generation micromechanical force (MMF) apparatus was used to observe and measure the cyclopentane film growth rate and hydrate interparticle cohesive force. The cohesive force measurement technique was adapted from the method reported by Aman et al. ${ }^{16,18}$ and studies of asphaltene cohesion performed by Morrissy et al. ${ }^{36}$ to which the reader is referred for further detail. The apparatus consisted of an Olympus IX-73 inverted light microscope (Figure 2) equipped with digital recording equipment, with images captured and analyzed via Olympus Stream Motion software.

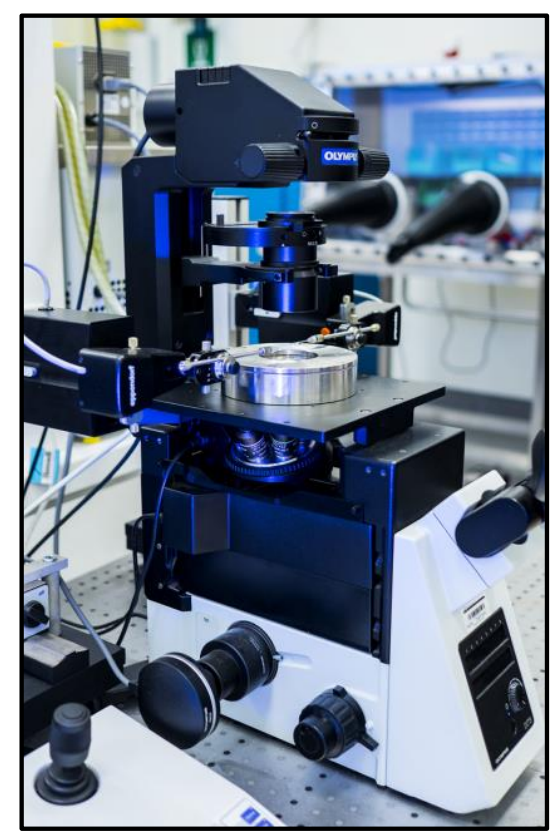

Figure 2. The micromechanical force apparatus consists of an inverted light microscope and experimental cell resting on an active pneumatic vibration isolation table.

An experimental cell was placed atop the microscope stage, and was surrounded by temperature-controlled aluminum jacket. A water-glycol mixture was circulated through the cooling jacket using a ThermoFisher cooling/heating bath $\left(-30\right.$ to $\left.80{ }^{\circ} \mathrm{C}\right)$, and the experimental cell was filled with liquid cyclopentane (> 99\% Sigma-Aldrich). The temperature of fluids in the cell was measured directly with a calibrated platinum resistance thermometer (PRT) with $0.01{ }^{\circ} \mathrm{C}$ resolution. The microscope and cell assembly were placed atop an active pneumatic vibration isolation table (Standa 1VIS10W) to minimize the effect of external forces on the hydrate particles. The cell contained two glass capillaries $(1000 \mu \mathrm{m}$ internal diameter) that were held by stainless steel arms (Eppendorf). A carbon-fiber filament (7 $\mu \mathrm{m}$ external diameter from Fiber Glast Developments Corporation) was secured within each capillary tube by epoxy adhesive. 
In each cohesive force experiment, a deionized water droplet was placed on the tip of each carbon-fiber cantilever, which was then quenched in liquid nitrogen to form ice. The ice particles were then placed in the liquid cyclopentane bath, which was maintained at $1{ }^{\circ} \mathrm{C}$; cyclopentane (structure II) hydrate grew at the water-cyclopentane interface immediately following ice dissociation. The hydrate particles were then allowed to anneal in the cyclopentane bath for at least 45 minutes prior to starting the experiment.

The cohesive force between both hydrate particles was measured indirectly using a four-step method described by Yang at al. ${ }^{37}$ (Figure 3): (i) the top particle was lowered into contact with the bottom particle, providing a preload displacement; (ii) the particles remained in contact for 10 seconds to reach mechanical equilibrium; (iii) the top particle was then raised at constant velocity until the particles separated; and (iv) the displacement was captured visually by the Olympus Stream Motion recording software.

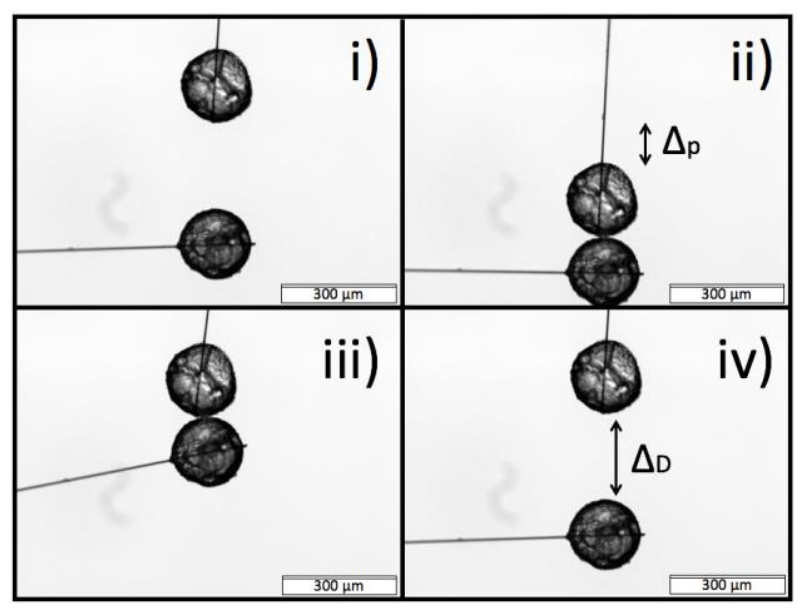

Figure 3. A four-step pull-off procedure allows for direct calculation of the preload and cohesive force as the product of the spring constant $\left(k_{\text {spring }}\right)$ and preload or cohesive displacements $\left(\Delta_{P}, \Delta_{D}\right)$, respectively.

The spring constant, $k$, of the carbon-fiber cantilever was calculated from the geometric and material properties of the fiber using equation ( 1 ):

$$
k=\frac{3 \pi E d^{4}}{64 L^{3}}
$$

where, $E$ is the elastic modulus of the material, $d$ is the diameter of the fiber, and $L$ is the length of the fiber. The elastic modulus, fiber diameter, and fiber length had relative 
uncertainties of $6 \%, 7 \%$, and $0.1 \%$, respectively. The elastic modulus of the carbon fiber was reported by Ilankeeran et al. ${ }^{38}$ as $250 \mathrm{GPa}$. Typically, carbon fibers lengths were between 3000 and $4000 \mu \mathrm{m}$, and were measured to within $2 \mu \mathrm{m}$ using the microscope. Morrissy et al. ${ }^{36}$ benchmarked values of carbon fiber spring constants determined from eq (1) against that of a reference tungsten wire, and found consistency within the estimated experimental uncertainty.

The spring constant of the carbon fiber was used to determine the inter-particle force at cohesive failure from the measured lateral displacement using Hooke's Law. To compare the results for particles of different sizes, the measured cohesive force was divided by the harmonic mean radius of the particle pair ( $\mathrm{R}^{*}$ in eq $\left.(2)\right)$. In each experiment, at least ten pull-off trials were performed to obtain a statistically-representative sample.

$$
R^{*}=\frac{2 R_{1} R_{2}}{R_{1}+R_{2}}
$$

\subsection{Hydrate Film Growth Rate}

The MMF apparatus setup was modified to allow measurements of the linear growth rate of cyclopentane hydrate film. In each growth rate experiment, one hydrate particle was created on the bottom cantilever (Figure 4) and placed in the liquid cyclopentane bath. After this, a deionized water droplet was placed on the top cantilever, and then submerged into the liquid cyclopentane. The water droplet was brought into contact with the hydrate particle, from which point the growth of a hydrate film was timed.

As shown in Figure 3b, hydrate was observed to grow along the water-cyclopentane interface after the water droplet was brought into contact with the hydrate particle. As the induction time for cyclopentane hydrate exceeds 24 hours under these moderate $\left(6.5^{\circ} \mathrm{C}\right)$ subcoolings, the measured hydrate growth along the interface was assumed to be triggered by the crystal template of the bottom particle and not caused by independent nucleation at other sites on the interface (which was not observed). In each experiment, the position of the growing hydrate interface was analyzed as a function of time to estimate the linear growth rate of the film using eq (3). 

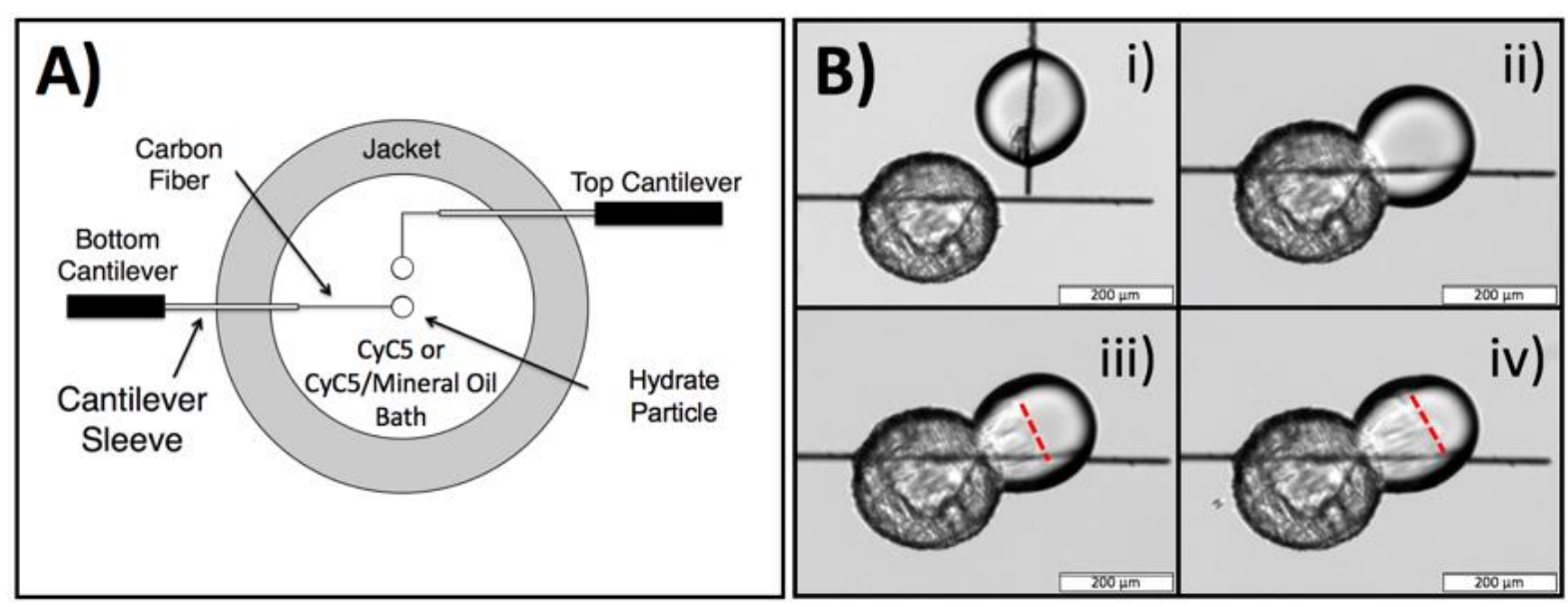

Figure 4. A) Top view schematic of experimental cell placed atop inverted light microscope where (B) a four step film growth procedure, where the advancing film position is demarcated by a dashed red line.

$$
F G R=\frac{\sqrt{\left(y_{2}-y_{1}\right)^{2}+\left(x_{2}-x_{1}\right)^{2}}}{t_{2}-t_{1}}
$$

Here $F G R$ is the linear film growth rate, $x_{l}$ and $y_{1}$ are the horizontal and vertical distances from origin at time $t_{1}$, and $x_{2}$ and $y_{2}$ are the horizontal and vertical distances from origin at time $t_{2}$. Measurements were collected until the hydrate film was observed to cover the entire water droplet surface. As this measurement is performed in two dimensions, it does not account for spatial variations in hydrate film thickness, which was assumed to be homogenous for this analysis. The hydrate film growth rates reported here represent the average of all film growth rate measurements performed across repeat experiments; typically between 5 and 10 repeat trials were conducted.

To investigate their impact on hydrate film growth rate, each of the surface-active oil subclasses identified in Section 2.1 was diluted in toluene, cooled in an ice bath, and injected into the liquid cyclopentane phase of the MMF cell which also contained the hydrate particle and water droplet of interest. The cell fluids were then mixed by hand for three minutes prior to performing the experiment, to facilitate sufficient adsorption of the surface active species to the hydrophilic-hydrophobic interfaces.

\section{$3 \quad$ Results and Discussion}

In each experiment, one existing hydrate particle was used as a crystal template to initiate the growth of a hydrate film in the water droplet, which was assumed to be independent of 
the morphological characteristics of the existing hydrate particle. To test this assumption, 18 independent measurements of hydrate film growth rate were performed in the absence of any chemical additive. Hydrate was only observed to grow at the water-cyclopentane interface over the timescale of the experiments, without changing the morphology of the hydrate particle interior. The resultant mean linear film growth rate was $2.2 \pm 0.2 \mu \mathrm{m} . \mathrm{s}^{-1}$, where the uncertainty bounds represents a $95 \%$ confidence interval. This result agreed well with preliminary measurements reported by Aman et al. ${ }^{11}$ for cyclopentane hydrate. To our knowledge, no other quantitative reports of cyclopentane hydrate film growth rates are available in the literature.

These measured growth rates for cyclopentane hydrate were compared with values predicted using the model developed by Mori $^{21}$ under the assumption of heat transport-limited hydrate growth. This model requires an estimate of the film's initial thickness, which we took from the literature. Taylor et al. ${ }^{23}$ reported that cyclopentane hydrate had an initial thickness of approximately $12 \mu \mathrm{m}$, which increased to approximately $35 \mu \mathrm{m}$ over the period of about 100 minutes. Haber et al. ${ }^{39}$ reported measurements of the thickness of cyclopentane hydrate shells 24 hours after formation of between 55 and $110 \mu \mathrm{m}$. For cyclopentane hydrate film thicknesses in the range of 10 to $55 \mu \mathrm{m}$, the heat-transfer limited model of Mori predicts growth rates of 16 to $65 \mu \mathrm{m} . \mathrm{s}^{-1}$. This is over an order of magnitude larger than the values observed here for cyclopentane hydrate, which suggests that either the film thickness is much greater than assumed (unlikely given the size of the water droplets used) or that heat transfer limitations do not dictate the rate of cyclopentane hydrate film growth. When compared to methane-water systems, the rate of film growth along the water-cyclopentane interface may be affected by variations in the thickness of the interface before or after growth.

\subsection{Crude Oil Fractions Limit Hydrate Growth}

Once the baseline film growth was established, the effect of free resins (FR), binding resins (BR), and residual asphaltenes (RA) on hydrate film growth rate was studied. The linear film growth rate was measured as a function of the "additive" mass fraction of each oil sub-class in the liquid cyclopentane phase at $1{ }^{\circ} \mathrm{C}$. The mass fraction of the additive was calculated gravimetrically based on mass fraction of the additive in toluene and the mass fraction of the dosed sample in the bulk phase. Together, the results (Figure 5) illustrate a clear trend in the 
order in which crude oil subclasses affect hydrate film growth rate: RAs > BRs > FRs from strongest to weakest.

With the addition of 4 ppm RA to the system, the rate of hydrate film growth decreased by one order of magnitude relative to its value at $2 \mathrm{ppm}$. As the RA mass fraction was further increased from 4 to $240 \mathrm{ppm}$, the rate of hydrate film growth decreased to within the measureable limit of the method ( $<1 \mu \mathrm{m}$ within a one-hour measurement period).

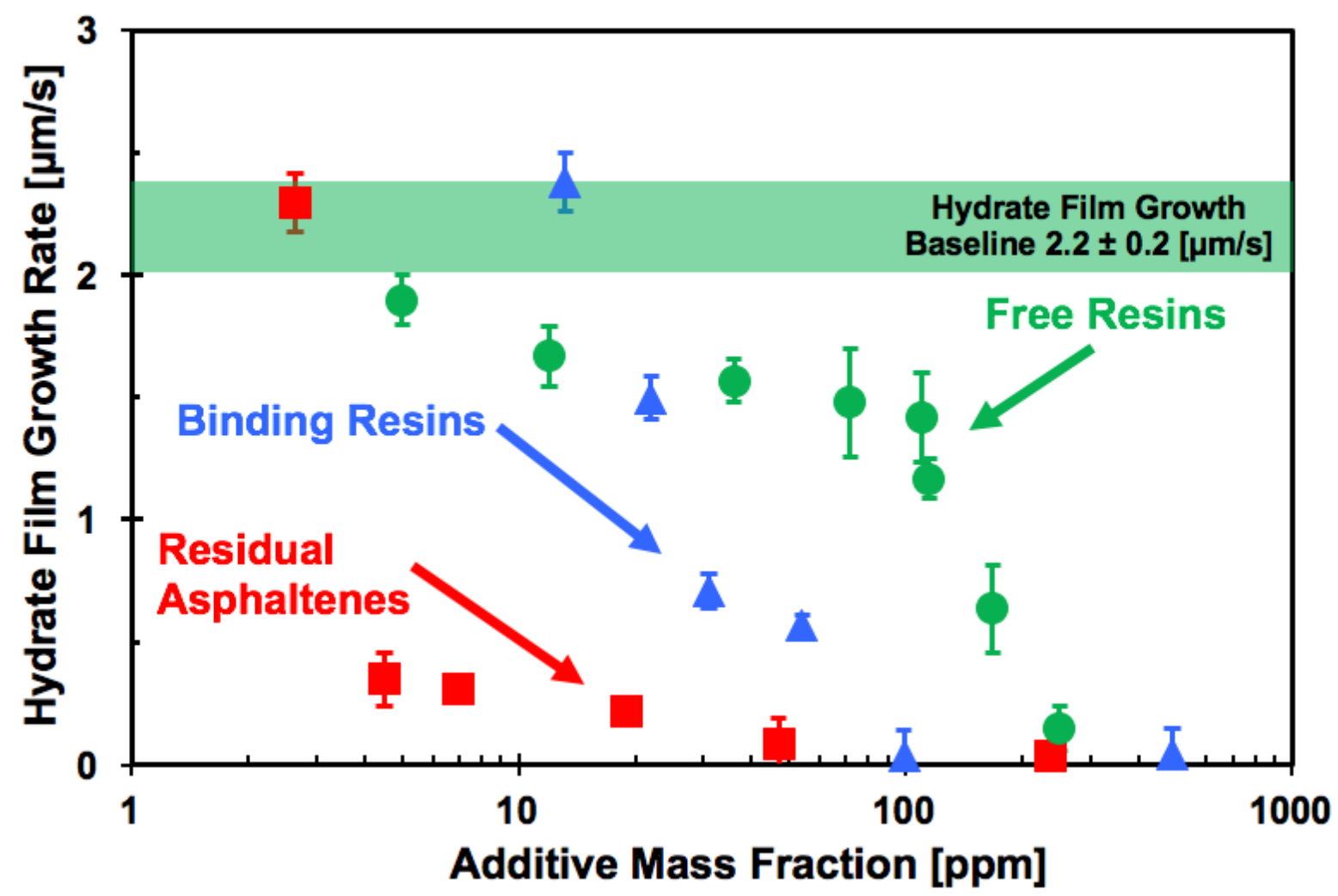

Figure 5. Estimated cyclopentane hydrate film growth rate as a function of mass fraction of residual asphaltenes (red squares), binding resins (blue triangles), and free resins (green circles) at $1{ }^{\circ} \mathrm{C}$, where each data point is the average of at least 10 independent film growth measurements and the uncertainty bounds represent a $95 \%$ confidence interval.

The result of adding BRs to the cyclopentane was similar to that observed for the RAs, although the amount of BR required to suppress hydrate film growth rate was an order of magnitude higher than that observed for the RA. In contrast, the injection of FRs into the cyclopentane had a limited effect on the hydrate film growth rate for mass fractions up to 100 ppm. Above this threshold, they were observed to sharply decrease the rate of hydrate film growth, to a level comparable with that observed for the RAs and BRs. 
Although surfactants may also interact with the three-phase boundary (water-cyclopentanehydrate), such adsorption may primarily affect the time delay observed before the hydrate film begins growing along the water-cyclopentane interface. The result shown in Figure 5 suggest that, above a given concentration threshold, each additive species may be adsorbing to the water-cyclopentane interface and disrupting assembly of the growing hydrate crystal. The energetic cost of the requisite desorption from the water-oil interface may manifest as a decreased apparent hydrate film growth rate. The low additive concentrations of RAs required to achieve the significant growth rate decrease is consistent with the fact that they are the most polar and aromatic components within a crude oil, and have with limited solubility in cyclopentane. The concentration of the RAs, BRs and FRs in the original oil were determined from the SARA fractionation to be 700, 500, and 2900, respectively. The naturally-occurring amount of each fraction was greater than the mass fraction required to suppress hydrate film growth below the detectable limit in the MMF.

While the precise distribution of molecular structure within the three subclasses has not been well-established, Graham et al. ${ }^{32}$ proposed that BR were less aromatic than RAs and may be constructed of saturated hydrocarbon chains with small aromatic moieties. Accordingly, BRs may be considered as more traditional surfactants than RAs, in that they have distinct lipophilic and hydrophilic moieties. When coupled with the absence of a large polynuclear aromatic core and lower molecular weight. From the SARA fractionation process, we know that BRs are more soluble in light alkanes than the RAs since they are extracted from the RAs with hot heptane. We hypothesize that this increased solubility is responsible for the shift in mass fraction when comparing RAs and BRs (Figures 5 and 6, respectively). The FRs may have the most traditional surfactant-like structure of the three subclasses studied, where these contain saturated hydrocarbon chains with traditional heteroatoms: sulfur, nitrogen, and oxygen. While it is clear the FRs are also effective at inhibiting growth the improved solubility of the FRs in the light alkanes (evidenced by the solubility in ambient temperature light alkanes during the SARA fractionation) relative to the BRs is likely to be responsible for the higher concentration at which this effect is observed.

The results illustrate that all three crude oil subclasses studied decrease the growth rate of a hydrate film by more than $99 \%$ at the highest concentrations tested. We infer from these results that the aromaticity of the additive contributes to its adsorption potential at the wateroil interface, where polynuclear aromatic species may also be able to utilize $\pi$ - $\pi$ stacking at 
the interface to achieve greater adsorption density. This hypothesis could possibly be tested through the use of molecular dynamics simulation, or by comparing experimental results obtained for this crude oil against those of other oils with varying degrees of reservoir biodegradation. We note, however, that the hydrocarbon liquid phase used in these measurements (cyclopentane) does not represent the chemical diverse crude oils present in pipelines. Accordingly, any up-scaling of these bench-top results must also consider whether other components present in the particular crude oil may limit adsorption capacity at the water-oil interface.

\subsection{Crude Oil Fractions Decrease Hydrate Cohesive Force}

While measuring hydrate film growth rate, it was possible to also observe the contact between the water droplet and hydrate particle. Together with the cohesive force measurement capability of the MMF, this provided a further opportunity to investigate whether each crude oil subclass affected the water wetting angle on the hydrate surface. As the mass fraction of each additive was increased from 5 to $250 \mathrm{ppm}$, the hydrate surface transitioned from water-wet (top row of Figure 6) to oil-wet (bottom row of Figure 6). This suggests that the surface active components within the crude oil subclasses adsorbed to both the hydrate-oil and water-oil interfaces; through Young's equation, it can be shown that a surface cannot transition from water-wet to oil-wet if only one of the three interfacial tensions in the system is modified. In the case of the RA at $250 \mathrm{ppm}$, the water droplet had to be held manually next to the hydrate particle, which otherwise repelled contact with the water droplet. In the context of oil and gas flowlines, this result suggests that the wettability of hydrate particles, and their aggregation or deposition potential by extension, can vary significantly with the chemical diversity of the suspending fluid; that is, the classical assumption of water-wet hydrate particles may not be appropriate for flowlines containing biodegraded crude oils. Given that these wetting images (Figure 6) were acquired prior to the hydrate film growth measurements, the results further illustrate that hydrate growth can occur along a water-oil interface even in the limit that the hydrate particle is apparently oil-wet. The comparison of wetting and film growth behavior further illustrates an underlying objective of future work, in utilizing water-oil interfacial tension data to predict hydrate film growth rate. This observation may be the consequence of long-range water ordering caused by templating of the hydrate crystal structure; this hypothesis could also potentially be tested with molecular dynamics simulation. 


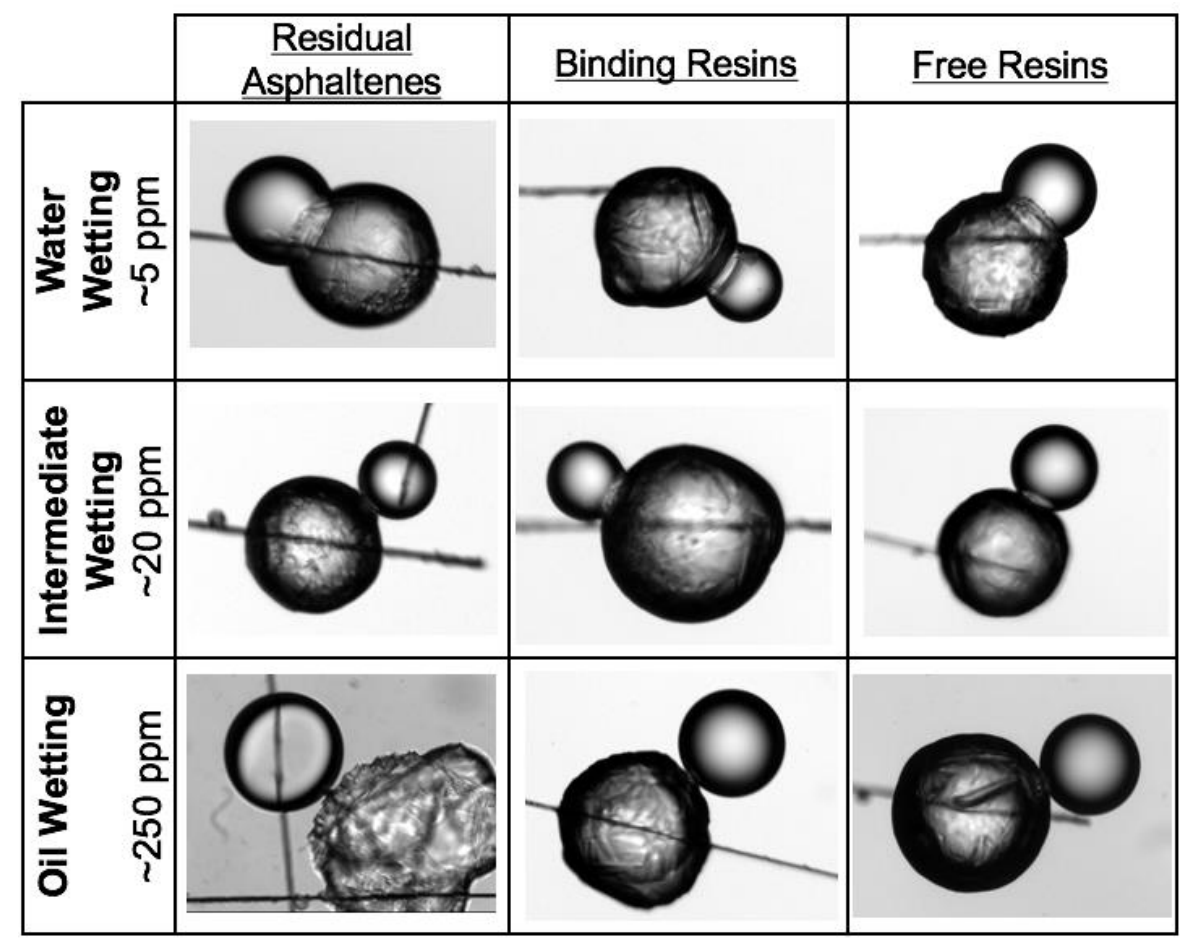

Figure 6. Photomicrographs of a deionized water droplet during first contact with a cyclopentane hydrate particle, as a function of the additive mass fraction in the liquid hydrocarbon phase.

As the cohesive force between hydrate particles is a function of both the water-oil interfacial tension and hydrate surface wettability, the above observations suggested that the addition of each crude oil subclass to the oil phase should result in a reduction of hydrate cohesive force. This was confirmed by conducting MMF cohesive force measurements for each subclass at the respective mass fractions that minimized hydrate film growth rate $\left(\leq 0.04 \mu \mathrm{m} . \mathrm{s}^{-1}\right.$ as shown in Figure 5). The hydrate cohesive force experiment was conducted independently of any film growth trial; two new hydrate particles were generated for each cohesive force trial. The results (Figure 7) illustrate that, at these relatively high mass fractions, each of the crude oil subclasses was able to suppress hydrate cohesive force by at least two orders of magnitude. The current generation of industrial AAs typically use quaternary ammonium salts, ${ }^{26,28}$ with the ionic surfactant head group adsorbing to the hydrate surface. Observations by Graham et al. ${ }^{32}$ on the general structure of RAs, BRs, and FRs suggests that these naturally-occurring surfactants are very different to industrial AAs, with these natural species typically having significantly higher molecular weights, nonionic head groups, and polyaromatic moieties. 


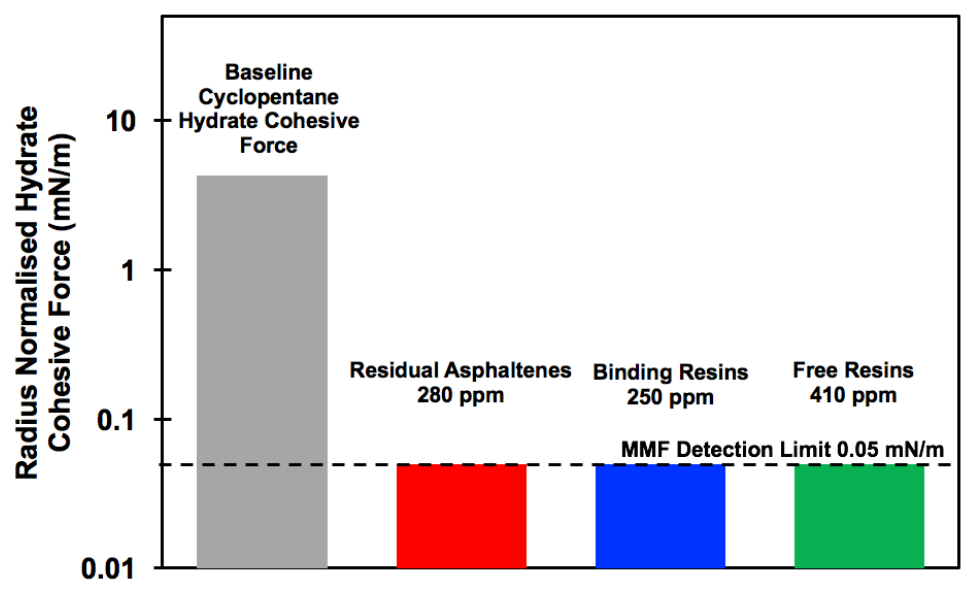

Figure 7. Hydrate cohesive force normalized to the particle radius for each of the crude oil subclasses at $1^{\circ} \mathrm{C}$.

While this study has considered the effect of each additive on hydrate growth and wettability, additional work is required to characterize the combined effect of these constituent groups. As a first approximation, we hypothesize that these constituents may be less effective in combination than in isolation, due to both (i) potential cross-interactions between constituent groups, enhancing solubility in the oil phase, and (ii) competition between surfactants for limited interfacial area.

\section{Conclusions}

In this study, we present the first systematic study of cyclopentane hydrate film growth rate as a function of various additive concentrations of the component sub-classes in an Australian crude oil., Relative to the baseline growth rate was established at $2.2 \pm 0.2 \mu \mathrm{m} . \mathrm{s}^{-1}$, the effect of free resins, binding resins, and residual asphaltenes was studied on hydrate film growth rate, hydrate particle wettability, and hydrate cohesive force using a micromechanical force apparatus. To reduce the hydrate film growth rate below $0.5 \mu \mathrm{m} . \mathrm{s}^{-1}, 4 \mathrm{ppm}$ of the residual asphaltene fraction was required, compared to $100 \mathrm{ppm}$ of the binding resins and $250 \mathrm{ppm}$ of the free resins. At sufficient concentrations, each subclass was also observed to invoke a transition in the hydrate particle surface from moderately water wet to fully oil wet. Interestingly, a hydrate film was still observed to grow along the water-oil interface in the limit of an oil-wet hydrate particle even when the hydrate particle repelled contact with the water droplet; to our knowledge of the literature, this behaviour has not been reported to date, and may be the consequence of long-range interaction between the existing hydrate crystal and the virgin water-oil interface. Finally, all three subclasses were able to reduce hydrate 
cohesive force below the detectable limit of the apparatus $(0.05 \mathrm{mN} / \mathrm{m})$, which is 1000 times below the average cohesive force suggested by Sinquin et al. ${ }^{40}$ from flowloop and rheology investigations. Together, the results illustrate that these subclasses may be active at both the hydrate-oil and water-oil interfaces, and potentially provide a mechanism to characterize or control the aggregation or deposition of hydrate particles in subsea hydrocarbon flowlines. However, further testing of these observations and inferences are required because the hydrocarbon liquid phase considered in this work was very different to that present in crude oil pipelines, where these subclasses may exist in abundance. Future work will analyze the mass fraction dependence of each subclass, and compare subclasses across differentlybiodegraded crude oils.

\section{Corresponding Author}

* Corresponding author: zachary.aman@uwa.edu.au, +61 864883078

\section{Acknowledgments}

This work was funded through the ARC Training Centre for LNG Futures (IC150100019).

The MMF apparatus was made available as part of the National Geosequestration Laboratory, funded by the Australian Government through the Education Investment Fund.

\section{Reference}

(1) Sloan, E. D.; Koh, C. A. Clathrate Hydrates of Natural Gas; 3 ed.; CRC Press 2007.

(2) Koh, C. A.; Sloan, E. D.; Sum, A. K.; Wu, D. T. Fundamentals and Applications of Gas Hydrates. Annual Review of Chemical and Biomolecular Engineering 2011, 2 (1), $237-$ 257.

(3) Creek, J. L. Efficient Hydrate Plug Prevention. Energy \& Fuels 2012, 26 (7), 41124116.

(4) Boxall, J. A. N., S.H.; Aman, Z.M.; Norris, B.E.; Hughes, T.J.; Ioannou, K.; May, E.F. Hydrate Plug Dissociation via Active Heating: Uniform Heating and a Simple Predictive Model. Energy \& Fuels In Press 2016.

(5) Sloan, E. D. Hydrate Engineering; SPE Monograph Volume 21; Society of Petroleum Engineers: Richardson, Texas2000.

(6) Kvenvolden, K. A. A review of the geochemistry of methane in natural gas hydrate. Organic Geochemistry 1995, 23 (11-12), 997-1008. 
(7) Demirbas, A. Methane hydrates as potential energy resource: Part 1 - Importance, resource and recovery facilities. Energy Conversion and Management 2010, 51 (7), 1547 1561.

(8) Waite, W. F.; Santamarina, J. C.; Cortes, D. D.; Dugan, B.; Espinoza, D. N.; Germaine, J.; Jang, J.; Jung, J. W.; Kneafsey, T. J.; Shin, H.; Soga, K.; Winters, W. J.; Yun, T. S. Physical properties of hydrate-bearing sediments. Reviews of Geophysics 2009, 47 (4), RG4003.

(9) Chong, Z. R.; Yang, S. H. B.; Babu, P.; Linga, P.; Li, X.-S. Review of natural gas hydrates as an energy resource: Prospects and challenges. Applied Energy 2016, 162 (C), $1633-1652$.

(10) Folger, P. Gas hydrates: Resource and hazard. In Energy Policies and Issues, 2011, pp 97-106.

(11) Aman, Z. M.; Koh, C. A. Interfacial phenomena in gas hydrate systems. Chemical Society Reviews 2016, 45 (6), 1678-1690.

(12) Vysniauskas, A.; Bishnoi, P. R. A Kinetic Study of Methane Hydrate Formation. Chemical Engineering Science 1983, 38 (7), 1061-1072.

(13) Vysniauskas, A.; Bishnoi, P. R. Kinetics of Ethane Hydrate Formation. Chemical Engineering Science 1985, 40 (2), 299-303.

(14) Englezos, P.; Kalogerakis, N.; Dholabhai, P. D.; Bishnoi, P. R. Kinetics of gas hydrate formation from mixtures of methane and ethane. Chemical Engineering Science 1987, 42 (11), 2659-2666.

(15) Turner, D.; Boxall, J.; Yang, S. Development of a hydrate kinetic model and its incorporation into the OLGA2000® transient multiphase flow simulator. In 5th International Conference on Gas Hydrates, 2005.

(16) Aman, Z. M.; Brown, E. P.; Sloan, E. D.; Sum, A. K.; Koh, C. A. Interfacial mechanisms governing cyclopentane clathrate hydrate adhesion/cohesion. Physical Chemistry Chemical Physics 2011, 13 (44), 19796.

(17) Rao, I.; Koh, C. A.; Sloan, E. D.; Sum, A. K. Gas Hydrate Deposition on a Cold Surface in Water-Saturated Gas Systems. Industrial \& Engineering Chemistry Research 2013, 52 (18), 6262-6269.

(18) Aman, Z. M.; Joshi, S. E.; Sloan, E. D.; Sum, A. K.; Koh, C. A. Micromechanical cohesion force measurements to determine cyclopentane hydrate interfacial properties. Journal of Colloid and Interface Science 2012, 376 (1), 283-288.

(19) Nicholas, J. W.; Dieker, L. E.; Sloan, E. D.; Koh, C. A. Assessing the feasibility of hydrate deposition on pipeline walls-Adhesion force measurements of clathrate hydrate particles on carbon steel. Journal of Colloid and Interface Science 2009, 331 (2), 322-328. (20) Uchida, T.; Ebinuma, T.; Kawabata, J. a. i.; Narita, H. Microscopic observations of formation processes of clathrate-hydrate films at an interface between water and carbon dioxide. Journal of Crystal Growth 1999, 204 (3), 348-356.

(21) Mori, Y. H. Estimating the thickness of hydrate films from their lateral growth rates: Application of a simplified heat transfer model. Journal of Crystal Growth 2001, 223 (1-2), 206-212.

(22) Uchida, T.; Ikeda, I. Y.; Takeya, S.; Ebinuma, T.; Nagao, J.; Narita, H. CO2 hydrate film formation at the boundary between $\mathrm{CO} 2$ and water: Effects of temperature, pressure and additives on the formation rate. Journal of Crystal Growth 2002, 237-239 (1-4 I), 383-387.

(23) Taylor, C. J.; Miller, K. T.; Koh, C. A.; Sloan Jr., E. D. Macroscopic investigation of hydrate film growth at the hydrocarbon/water interface. Chemical Engineering Science 2007, $62(23), 6524-6533$. 
(24) Saito, K.; Sum, A. K.; Ohmura, R. Correlation of hydrate-film growth rate at the guest/liquid-water interface to mass transfer resistance. Industrial \& Engineering Chemistry Research 2010, 49 (15), 7102-7103.

(25) Wu, R.; Kozielski, K. A.; Hartley, P. G.; May, E. F.; Boxall, J.; Maeda, N. MethanePropane Mixed Gas Hydrate Film Growth on the Surface of Water and Luvicap EG Solutions. Energy \&amp; Fuels 2013, 27 (5), 2548-2554.

(26) Kelland, M. History of the Development of Low Dosage Hydrate Inhibitors. Energy \& Fuels 2006, 20 (3), 825-847.

(27) Wu, R.; Aman, Z. M.; May, E. F.; Kozielski, K. A.; Hartley, P. G.; Maeda, N.; Sum, A. K. Effect of Kinetic Hydrate Inhibitor Polyvinylcaprolactam on Cyclopentane Hydrate Cohesion Forces and Growth. Energy \& Fuels 2014, 28 (6), 3632-3637.

(28) Kelland, M. A. Production Chemicals for the Oil and Gas Industry; 2 ed.; CRC Press2014.

(29) Zhong, Y.; Rogers, R. E. Surfactant effects on gas hydrate formation. Chemical Engineering Science 2000, 55 (19), 4175-4187.

(30) Di Profio, P.; Arca, S.; Germani, R.; Savelli, G. Surfactant promoting effects on clathrate hydrate formation: Are micelles really involved? Chemical Engineering Science 2005, 60 (15), 4141-4145.

(31) Di Crescenzo, A.; Di Profio, P.; Siani, G.; Zappacosta, R.; Fontana, A. Optimizing the Interactions of Surfactants with Graphitic Surfaces and Clathrate Hydrates. Langmuir 2016, 32 (26), 6559-6570.

(32) Graham, B. F.; May, E. F.; Trengove, R. D. Emulsion inhibiting components in crude oils. Energy \& Fuels 2008, 22 (2), 1093-1099.

(33) Ghloum, E. F.; Oskui, G. P. Investigation of Asphaltene Precipitation Process for Kuwaiti Reservoir. Petroleum Science and Technology 2004, 22 (7-8), 1097-1117.

(34) Fridjonsson, E. O.; Graham, B. F.; Akhfash, M.; May, E. F.; Johns, M. L. Optimized droplet sizing of water-in-crude oil emulsions using nuclear magnetic resonance. Energy and Fuels 2014, 28 (3), 1756-1764.

(35) Aman, Z. M.; Olcott, K.; Pfeiffer, K.; Sloan, E. D.; Sum, A. K.; Koh, C. A. Surfactant Adsorption and Interfacial Tension Investigations on Cyclopentane Hydrate. In Langmuir, 2013; Vol. 29, pp 2676-2682.

(36) Morrissy, S. A.; Lim, V. W.; May, E. F.; Johns, M. L.; Aman, Z. M.; Graham, B. F. Micromechanical Cohesive Force Measurements between Precipitated Asphaltene Solids and Cyclopentane Hydrates. Energy \& Fuels 2015, 29 (10), 6277-6285.

(37) Yang, S.-o.; Kleehammer, D. M.; Huo, Z.; Sloan, E. D.; Miller, K. T. Temperature dependence of particle-particle adherence forces in ice and clathrate hydrates. Journal of Colloid and Interface Science 2004, 277 (2), 335-341.

(38) Ilankeeran, P. K.; Mohite, P. M.; Kamle, S. Axial Tensile Testing of Single Fibres. Modern Mechanical Engineering 2012, 02 (04), 151-156.

(39) Haber, A.; Akhfash, M.; Loh, K.; Aman, Z. M.; Fridjonsson, E. O.; May, E. F.; Johns, M. L. Monitoring of Hydrate Shell Growth using NMR Techniques. Langmuir 2015, 31 (32), 8786-8794.

(40) Sinquin, A.; Palermo, T.; Peysson, Y. Rheological and Flow Properties of Gas Hydrate Suspensions. Oil \& Gas Science and Technology 2004, 59 (1), 41-57. 